\title{
Ultrafast Relaxation Dynamics of Osmium-Polypyridine Complexes in Solution
}

\author{
Olivier Bräm, ${ }^{\dagger}$ Fabrizio Messina, ${ }^{\perp, \dagger}$ Etienne Baranoff, ${ }^{\ddagger}, \S$ Andrea Cannizzo, ${ }^{\dagger, \|}$ \\ Mohammad Khaja Nazeeruddin, ${ }^{\ddagger}$ and Majed Chergui*, $\dagger$ \\ ${ }^{\dagger}$ Laboratoire de Spectroscopie Ultrarapide and ${ }^{\ddagger}$ Laboratoire de Photonique et Interfaces, Ecole Polytechnique Fédérale de Lausanne, \\ ISIC, FSB, CH-1015 Lausanne, Switzerland \\ ${ }^{\S}$ School of Chemistry, University of Birmingham, Edgbaston, Birmingham B15 2TT, United Kingdom \\ "Institute of Applied Physics, University of Bern, Sidlerstrasse 5, CH-3012 Bern, Switzerland
}

ABSTRACT: We present steady-state absorption and emission spectroscopy and femtosecond broadband photoluminescence up-conversion spectroscopy studies of the electronic relaxation of $\mathrm{Os}(\mathrm{dmbp})_{3}(\mathrm{Os1})$ and $\mathrm{Os}(\mathrm{bpy})_{2}(\mathrm{dpp})(\mathbf{O s 2})$ in ethanol, where dmbp is $4,4^{\prime}$-dimethyl-2,2'-biypridine, bpy is $2,2^{\prime}$-biypridine, and dpp is 2,3 dipyridyl pyrazine. In both cases, the steady-state phosphorescence is due to the lowest ${ }^{3}$ MLCT state, whose quantum yield we estimate to be $\leq 5.0 \times 10^{-3}$. For Os1, the steady-state phosphorescence lifetime is 25 ns. In both complexes, the photoluminescence excitation spectra map the absorption spectrum, pointing to an excitation wavelength-independent quantum yield. The ultrafast studies revealed a short-lived

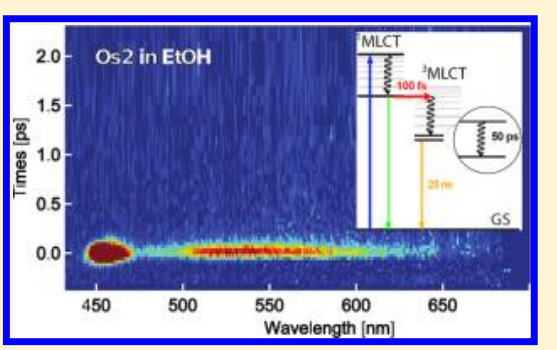
( $\leq 100 \mathrm{fs}$ ) fluorescence, which stems from the lowest singlet metal-to-ligand-charge-transfer $\left({ }^{1} \mathrm{MLCT}\right)$ state and decays by intersystem crossing to the manifold of ${ }^{3} \mathrm{MLCT}$ states. In addition, Os1 exhibits a 50 ps lived emission from an intermediate triplet state at an energy $\sim 2000 \mathrm{~cm}^{-1}$ above that of the long-lived (25 ns) phosphorescence. In Os2, the ${ }^{1} \mathrm{MLCT}-{ }^{3} \mathrm{MLCT}$ intersystem crossing is faster than that in Os1, and no emission from triplet states is observed other than the lowest one. These observations are attributed to a higher density of states or a smaller energy spacing between them compared with Os1. They highlight the importance of the energetics on the rate of intersystem crossing.

\section{INTRODUCTION}

Transition-metal (TM) complexes are attracting intense interest due to their applications in photocatalysis ${ }^{1}$ and solar energy conversion ${ }^{2}$ or as luminescent centers in organic lightemitting diodes (OLEDs) and light-emitting electrochemical cells (LEECs). ${ }^{3,4}$ In photocatalysis, it was found in the early 1970s that metal oxide semiconductors (SCs) such as titanium dioxide $\left(\mathrm{TiO}_{2}\right)$ could photoassist the electrochemical splitting of water or photoreduce $\mathrm{CO}_{2}$ to hydrocarbons. ${ }^{1,5}$ However, the band gap of $\mathrm{TiO}_{2}$ is at $3.2 \mathrm{eV}$, that is, in a spectral range where the solar spectrum is weak. To overcome this limitation, sensitization of the $\mathrm{TiO}_{2}$ substrate by adsorbed dye molecules allows injection of electrons into its conduction band (CB) upon excitation by visible light. This sensitization, initially aimed at extending to the visible range the photocatalytic applications, later became the basis of dye-sensitized-solar cells (DSSCs). ${ }^{6,7}$ Most DSSCs function with a TM dye complex that is adsorbed onto the surface of the SC. Upon light absorption by the complex, an electron is injected into the CB of the SC. Among the important factors determining the efficiency of DSSCs is the ability of the light-harvesting metal complex sensitizer to absorb photons in a broad visible-infrared range. ${ }^{7,8}$ So far, ruthenium-based polypyridine complexes are among the most used sensitizers. Their singlet metal-to-ligand chargetransfer $\left({ }^{1} \mathrm{MLCT}\right)$ excited states are the doorways to charge separation, and their maximum absorption lies in the green. Pushing further to the red the capabilities of sensitized $\mathrm{TiO}_{2}$ as photocatalysts or in DSSCs is highly desirable because it would allow exploiting a wider range of the solar spectrum.

Because of the above applications, TM-polypyridine complexes have attracted much interest from the community of ultrafast spectroscopists since the mid-1990s. ${ }^{9-19}$ The relaxation of the electronic states of metal-polypyridine complexes from and through the ${ }^{1} \mathrm{MLCT}$ states down to the triplet metal-to-ligand charge transfer $\left({ }^{3} \mathrm{MLCT}\right)$ states is characterized by an ultrafast cascade of internal conversion (IC), intramolecular vibrational redistribution (IVR), and intersystem crossing (ISC) events in tens to hundreds of femtoseconds. ${ }^{15,20,21}$ In sensitization by the commonly used $\mathrm{Ru}$-polypyridine complexes, electron injection is known to occur on two different time scales: a short one, from the initially excited nonthermalized ${ }^{1}$ MLCT state(s) within a few femtoseconds, ${ }^{22-24}$ and a slower one, from the thermalized ${ }^{3}$ MLCT states on the picosecond to nanosecond time scale. $^{25-28}$ The overall injection quantum yield is close to $100 \%$.

However, aside from ruthenium being a rare element, the spectrum of its polypyridine complexes is in the green, while, as previously mentioned, extending the absorption range further to the red would allow a better harvesting of the solar spectrum.

Received: May 31, 2013

Revised: July 6, 2013

Published: July 11, 2013 


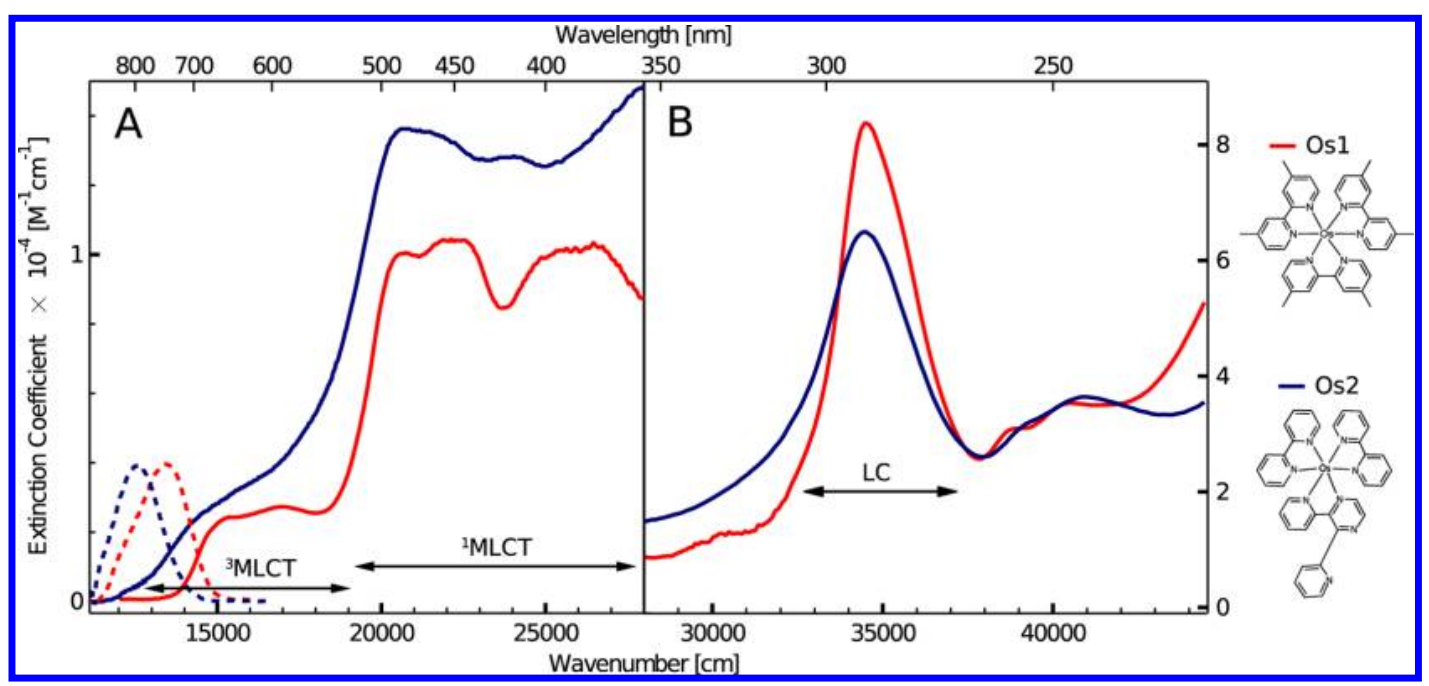

Figure 1. Extinction coefficient (continuous lines) in the 360-900 nm region (A) and in the $220-350 \mathrm{~nm}$ region (B) and steady-state emission (excited at $480 \mathrm{~nm}$, dashed lines, panel A) spectra of Os2 (blue) and Os1 (red) in ethanol. The arrows below the absorption spectra describe the band assignment: triplet $\left({ }^{3} \mathrm{MLCT}\right)$ and singlet $\left({ }^{1} \mathrm{MLCT}\right)$ metal-to-ligand charge-transfer and ligand-centered (LC) transitions. The molecular structure of both complexes is shown on the right.

Recent works with osmium-based polypyridine complexes have reported incident photon-to-current conversion efficiency (IPCE) extending well into the near-infrared region. ${ }^{29-34}$ These complexes show lower energy MLCT transitions and the spin-forbidden transition to the ${ }^{3}$ MLCT state should, in principle, be enhanced due to the stronger spin-orbit coupling (SOC) compared with ruthenium-based complexes. Accordingly, suitably designed Os-polypyridine sensitizers that display a broader absorption spectrum than their ruthenium equivalents, ${ }^{35}$ should lead to a better photoelectrochemical energy conversion. However, understanding their ultrafast photophysics is important to estimate the proportion of injection from nonthermalized ${ }^{1} \mathrm{MLCT}$ state and thermalized ${ }^{3} \mathrm{MLCT}$ ones.

In OLEDs and LEECs applications, the electronic states responsible for the emission are singlet and triplet excitons formed by pairing of electrons and holes. When TM complexes are used, the internal electroluminescence efficiency can be as high as $100 \%$ because both singlet and triplet excitons ultimately lead to photon emission from the lowest triplet state. ${ }^{3,36}$ This is a consequence of the high spin-orbit coupling (SOC) due to the metal ion, as it leads to an efficient ISC from excited singlet to the emissive triplet (sub)-states and can induce at the same time high radiative rates from the lowest excited triplet state to the singlet ground state. Thus, fast ISC is desired for applications of TM complexes as luminescent materials.

In a recent review, ${ }^{37}$ we discussed that the ISC rates in TM complexes do not necessarily scale with the SOC constant of the metal atom. In fact, while $\mathrm{Fe}$ or $\mathrm{Ru}$ polypyridine complexes exhibited ISC times of $<30 \mathrm{fs},{ }^{15,20}$ diplatinum complexes had ISC times three orders of magnitude longer. ${ }^{38}$ An extreme case was also reported by Steffen et al. for rhodium-cyclopentadienes, where the singlet fluorescence was dominant as a result of an inefficient ISC to the triplet state. ${ }^{39}$ The picture that emerges from these observations is that the SOC constant is not the only important ingredient but that the density of states and the molecular structure need to be taken into account for a proper description of the ISC rates. The structural considerations stemmed from the fact that either a favorable crossing of singlet and triplet surfaces was needed for an efficient ISC or that this crossing had to be made favorable by a structural rearrangement of the system. The latter was suggested by our study of halogenated rhenium carbonyl complexes, whose ISC times increased in the sequence $\mathrm{Cl}-\mathrm{Br}-$ $\mathrm{I}^{17}$ and were found to scale linearly with the frequency of the Rhenium-halogen vibration. As part of our studies on ultrafast ISC in TM complexes and given its importance for the abovementioned applications, here we investigate the electronic relaxation of two representative osmium complexes by means of steady-state absorption and emission spectroscopy and femtosecond broadband photoluminescence up-conversion.

Previously, high-resolution spectroscopy of osmium polypyridine complexes doped into low-temperature solids provided a detailed description of the low-lying triplet states and of their zero-field splittings, ${ }^{3,4,40-43}$ but it is only recently that their ultrafast relaxation dynamics have been investigated, mainly in relation to the sensitization of $\mathrm{TiO}_{2} \cdot{ }^{31,32,44}$ Here, by ultrafast photoluminescence techniques, we investigate the intramolecular dynamics of the complexes $\mathrm{Os}(\mathrm{dmbp})_{3}(\mathrm{PF} 6)_{2}$ (Os1) and $\mathrm{Os}(\mathrm{bpy})_{2}(\mathrm{dpp})\left(\mathrm{PF}_{6}\right)_{2}$ (Os2) in solution, where $\mathrm{dmbp}$ is $4,4^{\prime}$-dimethyl-2,2'-biypridine, bpy is $2,2^{\prime}$-biypridine, and $\mathrm{dpp}$ is 2,3-dipyridyl pyrazine. (The corresponding molecular structures are shown in Figure 1.) Os1 is a trisbipyridine osmium complex with methyl substituents on the pyridines, thus constituting an intermediate chemical structure between the parent $\left[\mathrm{M}(\mathrm{bpy})_{3}\right]^{2+}$ and that optimized with carboxylate groups to bind onto a SC surface. It is therefore ideal for a comparison with the much studied [Ru(bpy $\left.)_{3}\right]^{2+11,15,21}$ and $\left[\mathrm{Fe}(\mathrm{bpy})_{3}\right]^{2+18,20,21}$ complexes. One bipyridine ligand of $\left[\mathrm{Os}(\mathrm{bpy})_{3}\right]^{2+}$ has been substituted with dpp to form Os2. This compound is also investigated here and compared with Os1 to evaluate the effect of this peripheral substitution on its ultrafast photophysics.

\section{EXPERIMENTAL SECTION}

Steady-state absorption and emission measurements were carried out on a Shimadzu UV-3600 spectrophotometer and a Shimadzu RF-5301PC spectrofluorimeter, respectively. The emission spectra were corrected for the spectral response and 
dispersion of the detection system. Emission quantum yields were estimated by comparing the integrated steady-state emission intensity with that of a reference dye (rhodamine 6G in ethanol, quantum yield 0.95 ) after scaling for the optical density of the two samples.

The experimental setup for the ultrafast photoluminescence studies is described in detail in refs $45-47$, and we just present its features of relevance here. In brief, the sample was excited at either $400 \mathrm{~nm}$ (typical pulse width $60 \mathrm{fs}$, power $80 \mathrm{~nJ} /$ pulse, focal spot $30 \mu \mathrm{m}$ (fwhm), repetition rate $150-250 \mathrm{kHz}$ ) or between 480 and $700 \mathrm{~nm}$ from an optical parametric amplifier (OPA) system (Coherent, OPA-9400, typical width $70 \mathrm{fs,}$ power $80 \mathrm{~nJ} /$ pulse, focal spot $30 \mu \mathrm{m}$ (fwhm), repetition rate $150 \mathrm{kHz}$ ). The emission, collected in forward-scattering geometry, was up-converted in a $250 \mathrm{~mm}$ thick $\beta$-barium borate $(\mathrm{BBO})$ crystal by mixing it with a gate pulse at $800 \mathrm{~nm}$. The up-converted signal was spatially filtered and detected with a spectrograph and a liquid- $\mathrm{N}_{2}$-cooled charge-coupled device (CCD) camera in polychromatic mode. Appropriate Schott filters (Schott Glass Technologies) were used to attenuate the remaining excitation light. This greatly improved the signal-tonoise ratio but limited the detectable spectral range to a region starting $30 \mathrm{~nm}$ after the excitation wavelength up to $700 \mathrm{~nm}$. The latter limit is due to a filter cutting the strong secondharmonic generation (SHG) signal generated by the gate beam in the sum-frequency BBO crystal. The detection window can be extended to $740 \mathrm{~nm}$ by stretching the gate pulses, doubling its temporal width, which decreases the SHG background by a factor of four, enabling us to remove the filter cutting this spectral region. The time resolution of the setup is $\sim 110 \mathrm{fs}$, as measured by the fwhm of a kinetic trace of the Raman line of $\mathrm{H}_{2} \mathrm{O}$.

The Os(dmbp) $)_{3}(\mathrm{PF} 6)_{2}$ (Os1) and Os(bpy $)_{2}(\mathrm{dpp})\left(\mathrm{PF}_{6}\right)_{2}$ (Os2) complexes were synthesized according to the procedures of refs 48 and 49 and dissolved in ethanol. The roomtemperature solutions were circulated in a $0.2 \mathrm{~mm}$ thick quartz flow-cell at a speed of $1 \mathrm{~m} / \mathrm{s}$ to avoid photodamage. With the above experimental conditions, the excitation pulses hit the same spot $\sim 8$ times. However, because the ground-state recovery is $\ll 5 \mu$ s, all excited molecules relax to the ground state between pulses. The concentration was chosen to have typically between 0.1 and $0.3 \mathrm{OD}$ at the excitation wavelength, that is, $\sim 1 \mathrm{mM}$. Typical data acquisition times for all upconversion measurements were on the order of $0.5 \mathrm{~h}$ for one scan, the scans were repeated up to 10 times and then averaged.

To reliably extract the spectral contribution of each time component, we performed a kinetic analysis (multiexponential global fit (GF)) of the kinetic traces obtained by a singular value decomposition (SVD) of the $2 \mathrm{D}$ time-wavelength plot of Os1 in EtOH. The fitting procedure was performed using the following function:

$$
\begin{aligned}
I= & \left\{A_{1} \cdot \mathrm{e}^{\left(-t / \tau_{1}\right)}+A_{2} \cdot \mathrm{e}^{\left(-t / \tau_{2}\right)}+A_{3} \cdot \mathrm{e}^{\left(-t / \tau_{3}\right)}\right\} \\
& \otimes \exp \left[-\left(\frac{t-t_{0}}{\Delta_{\text {IRF }} / 2 \sqrt{\ln 2}}\right)^{2}\right]
\end{aligned}
$$

in which we assume three characteristic times $\left(\tau_{1}, \tau_{2}\right.$, and $\left.\tau_{3}\right)$ for the emission decay. The Gaussian term describes the convolution with the instrument response function (IRF), where $\Delta_{\text {IRF }}$ and $t_{0}$ are its full width at half-maximum (fwhm) and the time zero, respectively. In the GF procedure, the time constants have been considered as common kinetic parameters for all kinetic eigenvectors, whereas the amplitudes $A_{1}, A_{2}$, and $A_{3}$ have been determined for each kinetic eigenvectors. The decay-associated spectra are then deduced using the following equation:

$$
\operatorname{DAS}\left(\tau_{i}\right)=\sum_{j} A_{i}^{j} \cdot E_{j} \cdot S_{j}
$$

where the $A_{i}^{j}$ correspond to the preexponentional factor of $\tau_{i}$ obtained for the kinetic eigenvector $j$, and $E_{j}$ is the eigenvalue related to the kinetic and spectral eigenvectors $K_{j}$ and $S_{j}$.

\section{RESULTS}

III.1. Steady-State Spectroscopy. Figure 1 shows the steady-state absorption and emission spectra of Os1 and Os2 in ethanol. Both complexes show an intense and narrow band below $300 \mathrm{~nm}$ corresponding to an intraligand transition. Additional bands appear in the $350-550 \mathrm{~nm}$ region and are due to absorption by ${ }^{1}$ MLCT states. Finally, some weaker transitions lying in the $550-700 \mathrm{~nm}$ region are attributed to ${ }^{3}$ MLCT transitions in similar complexes. (See also Figure 2 for

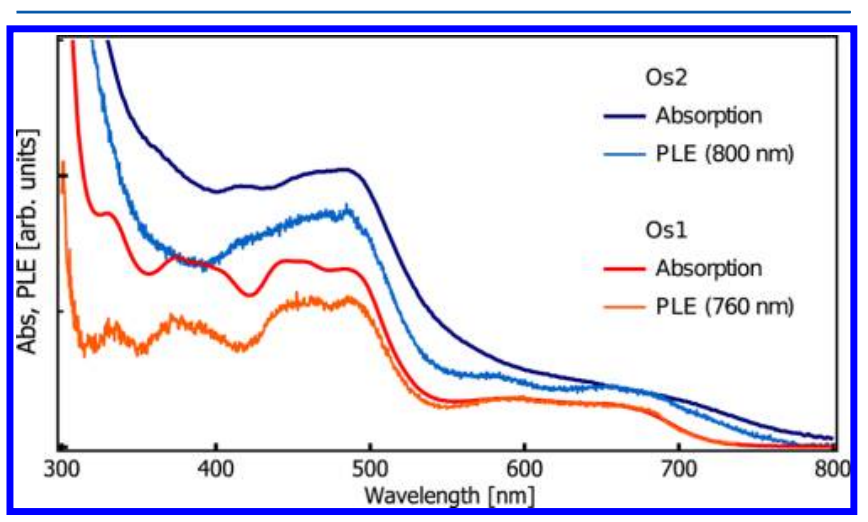

Figure 2. Static absorption and PLE of Os1 and Os2 in EtOH monitored at 760 and $800 \mathrm{~nm}$, respectively. The discrepancy in the short-wavelength range is due to the scattering background in the absorption.

an enhanced view of the 300 to $800 \mathrm{~nm}$ region.) ${ }^{43,50,51}$ Importantly, the integrated intensity of these ${ }^{3} \mathrm{MLCT}$ transitions with respect to that of the ${ }^{1} \mathrm{MLCT}$ states is larger than in the $\left[\mathrm{Ru}(\mathrm{bpy})_{3}\right]^{2+}$ or $\left[\mathrm{Fe}(\mathrm{bpy})_{3}\right]^{2+}$ complexes, hinting to a stronger mixed singlet-triplet character of the triplet state due to the larger SOC of osmium.

In the ${ }^{1}$ MLCT absorption region, we can distinguish two main group of bands, particularly clear in Os1, which are due to at least two different transitions. They exhibit structures at 485/ 440 and $410 / 370 \mathrm{~nm}$, whose spacings suggest a vibronic progression of $1600 \mathrm{~cm}^{-1}$ frequency, typical of the bpy stretch, already observed in $\left[\mathrm{M}(\mathrm{bpy})_{3}\right]^{2+}(\mathrm{M}=\mathrm{Fe}, \mathrm{Ru})$ complexes.

The ${ }^{3}$ MLCT manifold of states $(550-770 \mathrm{~nm})$ does not show two clear groups of bands as in the ${ }^{1} \mathrm{MLCT}$ region. Only one doublet of bands (at $\sim 590$ and $665 \mathrm{~nm}$ ) is observed. The energy spacing between them is $\sim 1600 \mathrm{~cm}^{-1}$, similar to that observed within a single group of bands in the ${ }^{1}$ MLCT transitions. One is then tempted to assign the $590 \mathrm{~nm}$ band to a higher vibronic band of the ${ }^{3} \mathrm{MLCT}$ transition having its origin at $\sim 665 \mathrm{~nm}$. The next vibronic transition would then be at $\sim 540 \mathrm{~nm}$, which is not clear in our spectrum. This interpretation also implies that the upper group of ${ }^{3} \mathrm{MLCT}$ transitions (if at all present) is shaded by the absorption of the 
${ }^{1}$ MLCT states in the $450-500 \mathrm{~nm}$ region. An alternative explanation for the doublet structure within the ${ }^{3} \mathrm{MLCT}$ manifold is that the two peaks at $\sim 590$ and $665 \mathrm{~nm}$ correspond to two different electronic states. The most likely interpretation at this stage is that both vibronic substructure and multiple electronic states contribute to the overall shape of the ${ }^{1,3} \mathrm{MLCT}$ absorptions, as witnessed by the additional features that show up clearly in both groups of the ${ }^{1}$ MLCT bands for Os1.

The absorption spectrum of Os2 grossly resembles that of Os1, but there are notable differences: (i) it is shifted to lower energies by $\sim 1000 \mathrm{~cm}^{-1}$, (ii) the bands therein are significantly broader, and (iii) the separation between different groups of bands is less clear cut. The latter two points suggest a higher density of states.

The steady-state emission is Stokes shifted by $\sim 2000 \mathrm{~cm}^{-1}$ with respect to the first absorption band with maxima at 740 and $795 \mathrm{~nm}$ for Os1 and Os2 in EtOH, respectively. In both cases, the emission spectra are broad and structureless. The emission intensity of both complexes is particularly weak compared with that of similar ruthenium complexes. Indeed, an estimate of the room-temperature quantum yield (QY) of emission upon $480 \mathrm{~nm}$ excitation (i.e., in the first ${ }^{1}$ MLCT transition), carried out as described in the Experimental Section, gives a value of $\sim 5 \times 10^{-3}$ for Os1 and $\sim 10^{-3}$ for Os2, while $\left[\mathrm{Ru}(\text { bpy })_{3}\right]^{2+}$ has a $\mathrm{QY} \sim 5 \times 10^{-2}$.

Figure 2 compares the absorption and the photoluminescence-excitation (PLE) spectra of Os1 and Os2, monitoring their emission intensity at 760 and $800 \mathrm{~nm}$, respectively. The increasing discrepancy between PLE and absorption spectra toward higher energies is due to the wavelength dependence of scattering that affects the absorption spectrum. Keeping this in mind, the PLE spectra match very well the absorption spectra, implying that the luminescence is not excitation-energydependent; that is, the relaxation from any initially excited electronic state cascades down to the lowest emitting triplet state, in agreement with the Kasha rule. This implies that the low-emission QYs of the lowest triplet state are due to its nonradiative decay to the ground state.

III.2. Time-Resolved Photoluminescence. Figure 3 shows the 2D time-wavelength plot of the early time luminescence of Os1 and Os2 in ethanol (EtOH) excited at $400 \mathrm{~nm}$. Both complexes exhibit a short-lived, almost responselimited, emission peaking at $550 \mathrm{~nm}$ for Os1 and $530 \mathrm{~nm}$ for Os2. Figure 4 shows the emission spectra of Os1 and Os2 in ethanol at $0,60,150$, and 300 fs (top panels) as well as kinetic traces at $530 \mathrm{~nm}(520 \mathrm{~nm})$ and $650 \mathrm{~nm}(600 \mathrm{~nm})$ for Os1 (Os2) in EtOH (bottom panels). The emission at early times appears mirror symmetric with respect to the lowest singlet absorption band, similar to our previous studies with other complexes; therefore, we attribute it to the singlet fluorescence. It mostly decays on an ultrafast time scale, as $\sim 90 \%$ of the signal at $530-550 \mathrm{~nm}$ is found to disappear within $\sim 100 \mathrm{fs}$ in both complexes.

In the case of Os1, in addition to the short-lived fluorescence at $550 \mathrm{~nm}$, we detect an obvious weak longer-lived emission centered at $650 \mathrm{~nm}$ (Figure 3). In these measurements, the spectral response rapidly drops beyond $700 \mathrm{~nm}$ due to the use of a band-pass filter in front of the monochromator (see Section II) so that a direct comparison with the steady-state emission is impossible. Thus, to make a connection of the present results on the femto-/picosecond regime with the static regime, we extended the detection window to the maximum of the steady-state emission maximum around $740 \mathrm{~nm}$ (see

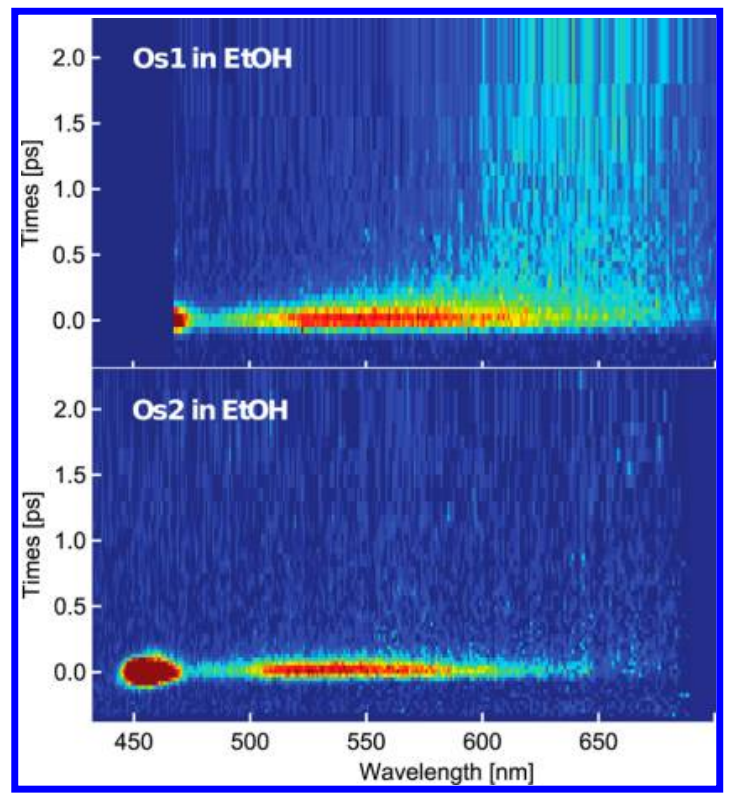

Figure 3. Two-dimensional time-wavelength plots of the emission of Os1 (top) and Os2 (bottom) in ethanol excited at $400 \mathrm{~nm}$. The plots are normalized to the maximum of the fluorescence, and the peak at $455 \mathrm{~nm}$ is the Raman line, which is cut on the blue by the detection window on the top panel.

Section II). Figure 5A compares the kinetic traces recorded at 730 and $700 \mathrm{~nm}$. Both traces are identical and are well-fitted by a monoexponential with a decay constant of $50 \pm 10 \mathrm{ps}$, meaning that the signal at $730 \mathrm{~nm}$ detected by fluorescence upconversion is only the extreme wing of a 50 ps lived band centered at $650 \mathrm{~nm}$ (Figure 3). We also measured the emission of Os1 in EtOH observed in the steady-state spectrum at 740 $\mathrm{nm}$ with a nanosecond resolution avalanche photodiode (Figure 5B), showing a time decay of $\sim 25$ ns (No such measurement was done for Os2). This finding implies that for Os1, the 50 ps lived signal revealed by ultrafast measurements has a different origin to the $25 \mathrm{~ns}$ lived emission detected in the static measurements, although the two signals partially overlap in the spectral region around $700 \mathrm{~nm}$. No picosecond-lived emission was observed in Os2.

III.3. Analysis. We performed an SVD of the fluorescence 2D plot of the two complexes in $\mathrm{EtOH}$ in terms of spectral and kinetic components before applying a global fitting procedure with exponential functions. ${ }^{17}$ The resulting decay-associated spectra (DAS) are plotted in panels A and C of Figure 6. The values of the decay constants are summarized in Table 1 . For Os1, this analysis reveals that besides two decay components of 100 fs and 50 ps with distinct spectral shapes (red and blue), a third component (green) of 1.5 ps appears as a decay around $580 \mathrm{~nm}$ and a rise around $660 \mathrm{~nm}$. This component can be interpreted as the singlet emission in the $550-620 \mathrm{~nm}$ region decaying in 1.5 ps and populating the 50 ps DAS, in agreement with the fact that the negative part of the 1.5 ps trace clearly resembles the 50 ps DAS (Figure 6). In the case of Os2, the intensity decay is biexponential with time constants of $<50 \mathrm{fs}$ and 150 fs. The corresponding DAS are similar to each other, although the longer component is slightly red-shifted, but the faster component has a 10-fold larger pre-exponential factor.

In summary, both Os1 and Os2 exhibit a broad emission band centered around 530-550 $\mathrm{nm}$ already at zero time delay (i.e., with a pulse-width-limited rise), which decays in $<50 \mathrm{fs}$ 


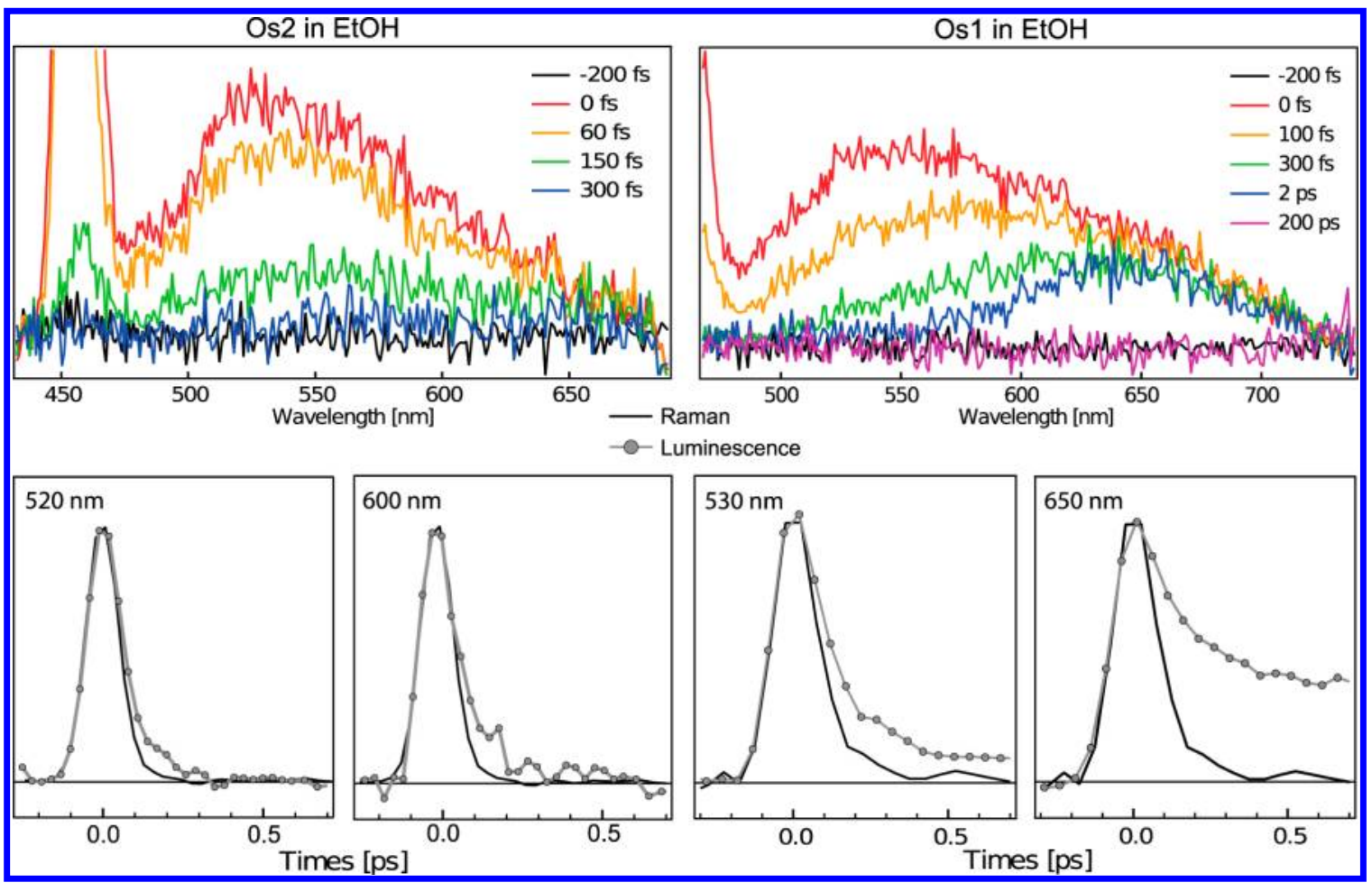

Figure 4. Top panels: time-gated fluorescence spectra of Os2 (left) and Os1 (right) in ethanol recorded at -200, 0, 60, 150, and 300 fs (in addition, 2 and 200 ps for N626) time delays. Bottom panels: kinetic traces (O) at 520 (530) and 600 (650) nm of Os2 (N626) in ethanol and DCM. For a better comparison of the kinetic behavior, the time traces are normalized to the maximum and plotted along with the kinetic trace (full) of the Raman line at $453 \mathrm{~nm}$.

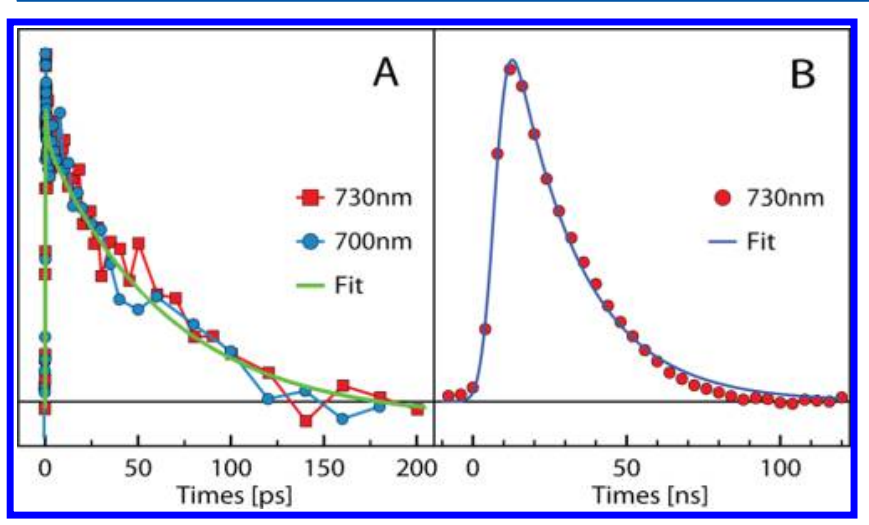

Figure 5. (A) Kinetic traces at 700 (blue circles) and $730 \mathrm{~nm}$ (red squares) of Os1 in ethanol by means of femtosecond up-conversion. For a better comparison of the kinetic behavior, the time traces are normalized to the maximum. A monoexponential fit of the trace at 730 $\mathrm{nm}$ is also shown (green line), which gives a decay time of $50 \pm 10 \mathrm{ps}$. (B) Kinetic trace at $730 \mathrm{~nm}$ of Os1 in ethanol (red circles), measured with a nanosecond photodiode. A monoexponential fit of the trace (blue line) gives a decay time of $25 \mathrm{~ns}$.

and $150 \mathrm{fs}$ for Os 2 and 100 fs and 1.5 ps for Os1. For the latter, the 1.5 ps time constant also corresponds to the rise of a longer-lived (50 ps) emission band centered at $650 \mathrm{~nm}$. Although partially overlapping with the $25 \mathrm{~ns}$-lived phosphorescence, the 50 ps emission is different from it (Figures 1 and 5). No comparable picosecond-lived emission is observed in Os2, where a fit of the integrated area of the signal (Figure 6C) shows the fluorescence to decay completely on the two time scales of $<50 \mathrm{fs}$ and $150 \mathrm{fs}$. Figure 7 shows a diagram summarizing the relaxation cascade for Os1. For the sake of simplicity, the $1.5 \mathrm{ps}$ intermediate is not shown.

\section{DISCUSSION}

Similar to the previously reported fluorescence of $\mathrm{Ru}$ - and Fepolypyridine complexes, ${ }^{15,20,21}$ the short-lived emission of Os complexes observed here corresponds to fluorescence of the lowest ${ }^{1}$ MLCT state. In both Os1 and Os2, this signal decays biexponentially. The faster, major decay is almost limited by our time resolution ( $\sim 50$ fs after deconvolution of the instrumental response) for Os2, while for Os1 it is slower $(\sim 100 \mathrm{fs})$. Moreover, in both complexes we observe a $\sim 10 \%$ contribution of a slower fluorescence decay of $1.5 \mathrm{ps}$ and $150 \mathrm{fs}$. By analogy to ruthenium complexes, such as $[\mathrm{Ru}-$ $\left.(\mathrm{dcbpyH})_{2}(\mathrm{NCS})_{2}\right]^{2+}(\mathrm{N} 719)^{21}{ }^{21}$ this could arise from the lower energy part of the ${ }^{1} \mathrm{MLCT}$ manifold of states undergoing slower ISC to the triplet manifold.

As mentioned in the Introduction, there is ample evidence ${ }^{17,21,37}$ that the ISC times generally do not scale with the strength of the spin-orbit constant of the metal atom and that the density of states, the molecular structure and the photoinduced structural dynamics play an important role in the ISC dynamics, providing, respectively, the system with a pool of accepting states or allowing it to reach a favorable molecular configuration for ISC to occur. In the present case, the two complexes have quite similar molecular structures, but their electronic structures are nonetheless significantly different (Figure 1), with Os2 having a higher density of states than Os1. This, along with the relative shifting of excited states, would be the origin of the faster intramolecular relaxation (IC, IVR, and ISC).

ISC in Os1 (on both $100 \mathrm{fs}$ and 1.5 ps time scales) populates a state that decays in 50 ps. Such a picosecond-decaying emission is absent in Os2. Because the 50 ps emission peaks around $\sim 650 \mathrm{~nm}$ (blue DAS in Figure 6), this implies that it 


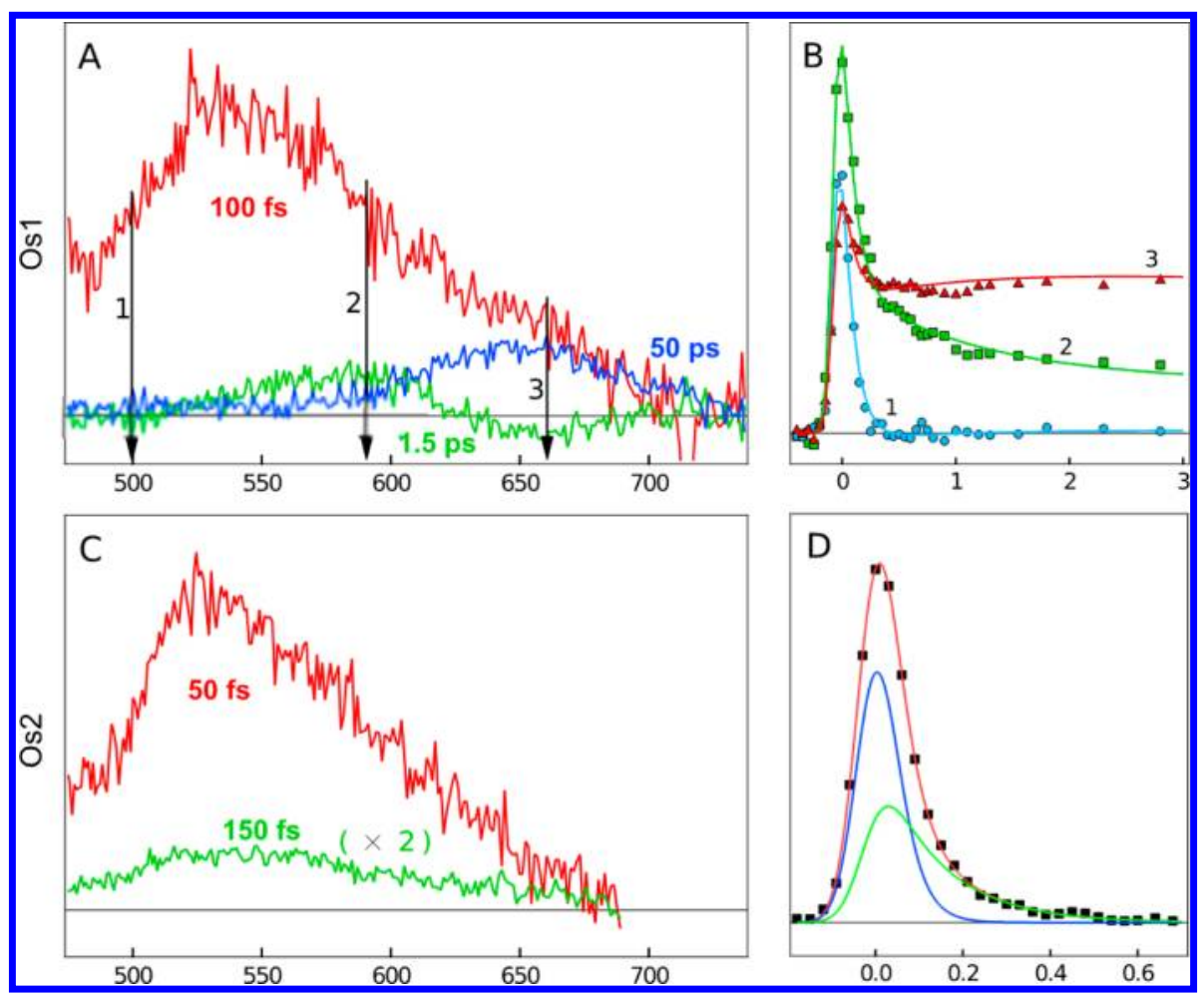

Figure 6. (A,C) Decay-associated spectra (pre-exponential factor of each time component as a function of wavelength) obtained with a global fit analysis of emission of Os1 (panel A) and Os2 (panel C). (The $150 \mathrm{fs}$ component of Os2 (green) is multiplied by two for clarity reasons.) (B) Kinetic traces at 500 (blue), 580 (green), and $660 \mathrm{~nm}$ (red) of the emission of Os2 along with their fit. (D) Integrated band area of the emission of Os2 as a function of time ( $)$ plotted along with its biexponential fit in red, decomposed into the blue and green lines corresponding to the $\sim 50 \mathrm{fs}$ and 150 fs components, respectively.

Table 1. Values of the Decay Constants Resulting from the Global Fit Analysis Presented in Figure 6

\begin{tabular}{cc} 
Os1 & Os2 \\
$100 \mathrm{fs}$ & $<50 \mathrm{fs}$ \\
$1.5 \mathrm{ps}$ & $150 \mathrm{fs}$ \\
$50 \mathrm{ps}$ & \\
\hline
\end{tabular}

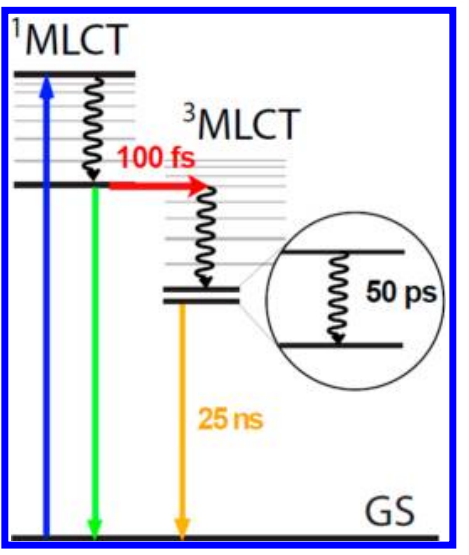

Figure 7. Relaxation cascade of Os1 as derived from the ultrafast photoluminescence studies.

does not stem from the lowest triplet state but from a higher lying state, which cannot be the ${ }^{1}$ MLCT. Assuming the absorption-emission Stokes shift (measured at the band maxima) to be identical to that of the ${ }^{1}$ MLCT emission, we estimate the 50 picosecond emission to stem from a state whose absorption band lies at $\sim 580 \mathrm{~nm}$. This corresponds to the upper band of the ${ }^{3}$ MLCT absorption doublet (Figures 1 and 2). It suggests that the spectral feature in the 550-700 nm region is not due to vibronic bands but to two different electronic states. By extrapolation, this would also confirm an analogous interpretation for the ${ }^{1} \mathrm{MLCT}$ absorption bands in the $430-550 \mathrm{~nm}$ and the $350-420 \mathrm{~nm}$ regions. Interestingly, the appearance of an intermediate ${ }^{3}$ MLCT emission on the time scale of tens of picoseconds is reminiscent of similar observations in the case of $\operatorname{ReX}($ bpy $)(\mathrm{CO})_{3}(\mathrm{X}=\mathrm{Cl}, \mathrm{Br}, \mathrm{I})$ complexes. ${ }^{17}$ The relaxation cascade that is inferred from the above and from Figure 2 means that close to $100 \%$ of the molecules in the lowest excited singlet state decay via this intermediate triplet state, in agreement with our conclusions based on the PLE spectra. Whereas a small portion of the $50 \mathrm{ps}$ emission rises in 1.5 ps (Figure 6), one would also expect to see it rising with a $100 \mathrm{fs}$ time scale. However, the absence of such a rise in the reddest portion of the 100 fs DAS is likely due to the broad overlapping profile of the $100 \mathrm{fs}$ decay of the ${ }^{1} \mathrm{MLCT}$ emission.

The 50 ps lived intermediate eventually populates the state responsible for the static phosphorescence, whose emission peaks at $\sim 750 \mathrm{~nm}$ (Figure 2) and decays in $25 \mathrm{~ns}$ (Figure 5B). On the basis of our estimate of the emission quantum yield, the latter has a radiative rate of a few microseconds $\left(\tau_{\mathrm{r}} \approx 0.025 / 5 \times\right.$ $\left.10^{-3}=5 \mu \mathrm{s}\right)$. Because the up-conversion signal is proportional to the radiative rate, this corresponds to a signal that is several hundred times weaker than that of the fluorescence (assuming a 10-20 ns lifetime for it) and thus below our detection limit. This explains why the steady-state phosphorescence is not observed in ultrafast measurements (i.e., no nanosecond-lived signal appears in Figure 5A, where only the 50 ps lived transient 
emission is detected). For comparison, in the case of $\left[\mathrm{Ru}(\mathrm{bpy})_{3}\right]^{2+15}$ which has a comparable radiative rate, the steady-state phosphorescence was close to the detection limit yet still measurable due to a better sensitivity of our detection system in the corresponding spectral region.

We have concluded above that the overall ${ }^{3}$ MLCT absorption (550-700 nm) contains (at least) two different states, the upper one being responsible for the 50 ps emission. In line with this, one would now be inclined to simply attribute the steady-state emission to the lower member of the doublet, that is, the lowest ${ }^{3} \mathrm{MLCT}$ state responsible for the absorption band peaking at $\sim 665 \mathrm{~nm}$. However, in high-resolution studies of $\left[\mathrm{Os}(\mathrm{bpy})_{3}\right]^{2+}$ at low temperatures, Yersin and coworkers $^{40,42,52}$ found that the lowest-lying triplet state is affected by an important zero-field splitting effect. It is actually split in three substates, with the lowest one (I) separated by 60 and $221 \mathrm{~cm}^{-1}$ from the next higher ones (respectively, II and III). While all three substates contribute to the lowest ${ }^{3}$ MLCT absorption band, at room temperature, the steady-state emission will mainly arise from substates I and II. The lifetime of substate I at $1.3 \mathrm{~K}$ was reported to be $22 \mu \mathrm{s}$, which is close to our estimated value of $5 \mu \mathrm{s}$, bearing in mind the uncertainties in the estimate of the QY. Yersin and coworkers also found a significant difference between the degree of allowedness of the transition from the ground singlet state to these triplet substates, with state I being the most forbidden, while states II and III become more allowed due to an increasing SOC to the higher singlet states. One may expect this trend to be carried over to the upper-lying ${ }^{3}$ MLCT states. Our results are in line with these suggestions. A radiative lifetime as long as $5 \mu \mathrm{s}$ implies that the transition responsible for the steady-state emission is very weakly allowed: based on the Strickler-Berg equation one can roughly estimate the peak molar extinction coefficient of this transition to be $\sim 130 \mathrm{M}^{-1} \mathrm{~cm}^{-1}$ (for each given non degenerate state, i.e., state I or II). Because this is well below the value $\left(2000 \mathrm{M}^{-1} \mathrm{~cm}^{-1}\right)$ measured in the ${ }^{3} \mathrm{MLCT}$ absorption region (Figure 1), we conclude that the state(s) responsible for the static emission does not contribute significantly to the overall absorption spectrum. The ${ }^{3} \mathrm{MLCT}$ absorption between 550 and $800 \mathrm{~nm}$ is actually due to upper ${ }^{3}$ MLCT states. On the basis of the comparatively higher oscillator strengths, these states are likely affected by a strong mixing with the ${ }^{1}$ MLCT states, which provides them with a significant singlet character, and hence a higher radiative rate, to the point that an intermediate 50 picosecond lived emission appears in the time-resolved luminescence spectra of Os1. The fact that such an emission is lacking in Os2 is, we believe, due to the same reasons as invoked above to explain the faster IC/ ISC processes: the density of vibrational states is higher or the energy spacing between electronic states is smaller, such that nonradiative relaxation is more efficient.

Coming back to considerations about sensitization and as discussed in ref 24 the ultrafast injection from nonthermalized ${ }^{1}$ MLCT states competes with an extremely high rate of IC/IVR. The latter has the additional consequence of lowering the energy of electronic states such that the driving force for electron injection decreases. Although Os2 has a large extinction coefficient extending to the red, which ensures a better collection efficiency of the solar spectrum, Os1 seems a more promising candidate for DSSCs because of the overall slower relaxation processes evidenced by the presence of excited states with lifetimes of 1.5 and $50 \mathrm{ps,} \mathrm{which} \mathrm{allows}$ ample time for injection, even with a low driving force. The faster relaxation processes in Os2, ensure a more rapid funnelling of the population to the lowest triplet state, and it is therefore a better candidate for OLEDs and LEECs.

Concerning the ISC rates, the present results are a further manifestation of the fact that a larger SOC constant of the metal atom (manifested by an increased triplet absorption), does not necessarily correlate with a shorter ISC time, as the present systems are quite close to the previously investigated $\left[\mathrm{M}(\mathrm{bpy})_{3}\right]^{2+}(\mathrm{M}=\mathrm{Fe}, \mathrm{Ru})$ complexes for which the metal atom has a smaller SOC constant. They further underline the importance of energetics in affecting the intramolecular relaxation processes, not only the ISC but also the IC and IVR processes. Indeed, small modifications of peripheral groups of the molecule lead to different ISC times $(<50$ fs for Os2 and $\sim 100$ fs for Os1). More studies are still needed to extract a rationale for the ISC rates in TM complexes.

\section{CONCLUSIONS}

We have investigated the intramolecular relaxation dynamics of two osmium complexes in solution: $\mathrm{Os}(\mathrm{dmbp})_{3}($ Os1) and $\mathrm{Os}(\mathrm{bpy})_{2}(\mathrm{dpp})$ (Os2). In both cases, the steady-state phosphorescence is due to the lowest ${ }^{3}$ MLCT state, whose quantum yield we estimate to $\leq 5.0 \times 10^{-3}$. The fluorescence of the lowest singlet metal-to-ligand-charge-transfer ( $\left.{ }^{1} \mathrm{MLCT}\right)$ state of both complexes was found to decay in $\leq 100$ fs due to an ISC to the manifold of ${ }^{3}$ MLCT states. Overall intramolecular relaxation (IC, ISC, and IVR) is faster in Os2 than in Os1, and the latter exhibits a 50 picosecond lived emission from an intermediate triplet state. Despite its better collection efficiency of the solar spectrum, Os2 is less attractive for applications using sensitization of $\mathrm{TiO}_{2}$ substrates. However, it is more interesting for applications such as light-emitting devices. From a fundamental point of view, these studies confirm previous observations that a large spin-orbit coupling of the metal atom is not necessarily accompanied by fast ISC rates. The present complexes highlight the importance of the density of states in affecting these rates.

\section{AUTHOR INFORMATION}

\section{Corresponding Author}

*E-mail: Majed.chergui@epfl.ch.

\section{Present Address}

${ }^{\perp}$ Fabrizio Messina: Dipartimento di Scienze Fisiche e Chimiche, Università degli Studi di Palermo, Via Archirafi 36, Palermo, Italy.

\section{Notes}

The authors declare no competing financial interest.

\section{REFERENCES}

(1) Linsebigler, A. L.; Lu, G. Q.; Yates, J. T. Photocatalysis on $\mathrm{TiO}_{2}$ Surfaces - Principles, Mechanisms, and Selected Results. Chem. Rev. 1995, 95, 735-758.

(2) Hagfeldt, A.; Gratzel, M. Molecular Photovoltaics. Acc. Chem. Res. 2000, 33, 269-277.

(3) Yersin, H. Triplet emitters for OLED Applications. Mechanisms of Exciton Trapping and Control of Emission Properties. Top. Curr. Chem. 2004, 241, 1-26.

(4) Yersin, H. Organometallic Triplet Emitters for OLED Applications. Controlling of Emission Properties by Chemical Variation. Proc. Soc. Photo-Opt. Instrum. Eng. 2004, 5214, 124-132.

(5) Fujishima, A.; Honda, K. Electrochemical Photolysis of Water at a Semiconductor Electrode. Nature 1972, 238, 37-38. 
(6) O'Regan, B.; Gratzel, M. A Low-Cost, High-Efficiency Solar-Cell Based on Dye-Sensitized Colloidal $\mathrm{TiO}_{2}$ Films. Nature 1991, 353, 737-740.

(7) Kalyanasundaram, K.; Gratzel, M. Applications of Functionalized Transition Metal Complexes in Photonic and Optoelectronic Devices. Coord. Chem. Rev. 1998, 177, 347-414.

(8) Nazeeruddin, M. K.; Baranoff, E.; Gratzel, M. Dye-Sensitized Solar Cells: A Brief Overview. Sol. Energy 2011, 85, 1172-1178.

(9) Damrauer, N. H.; Cerullo, G.; Yeh, A.; Boussie, T. R.; Shank, C. V.; McCusker, J. K. Femtosecond Dynamics of Excited-State Evolution in $[\mathrm{Ru}(\mathrm{bpy})(3)](2+)$. Science 1997, 275, 54-57.

(10) Yeh, A. T.; Shank, C. V.; McCusker, J. K. Ultrafast Electron Localization Dynamics Following Photo-Induced Charge Transfer. Science 2000, 289, 935-938.

(11) Bhasikuttan, A. C.; Suzuki, M.; Nakashima, S.; Okada, T. Ultrafast Fluorescence Detection in Tris (2,2 '-bipyridine)Ruthenium(II) Complex in Solution: Relaxation Dynamics Involving Higher Excited States. J. Am. Chem. Soc. 2002, 124, 8398-8405.

(12) Bhasikuttan, A. C.; Okada, T. Excited-State Relaxation Dynamics of $\mathrm{Ru}(\mathrm{dcbpy})(2)$ (NCS)(2), Studied by Fluorescence Upconversion Spectroscopy. J. Phys. Chem. B 2004, 108, 1262912632.

(13) Brady, C.; McGarvey, J. J.; McCusker, J. K.; Toftlund, H.; Hendrickson, D. N. Time-Resolved Relaxation Studies of Spin Crossover Systems in Solution. In Spin Crossover in Transition Metal Compounds III; Gütlich, P., Goodwin, H. A., Eds.; Springer: Berlin, 2004; Vol. 235, pp 1-22.

(14) Juban, E. A.; Smeigh, A. L.; Monat, J. E.; McCusker, J. K. Ultrafast Dynamics of Ligand-Field Excited States. Coord. Chem. Rev. 2006, 250, 1783-1791.

(15) Cannizzo, A.; van Mourik, F.; Gawelda, W.; Zgrablic, G.; Bressler, C.; Chergui, M. Broadband Femtosecond Fluorescence Spectroscopy of $[\mathrm{Ru}(\mathrm{bpy})(3)](2+)$. Angew. Chem., Int. Ed. 2006, 45, 3174-3176.

(16) Shaw, G. B.; Grant, C. D.; Shirota, H.; Castner, E. W.; Meyer, G. J.; Chen, L. X. Ultrafast Structural Rearrangements in the MLCT Excited State for Copper(I) Bis-Phenanthrolines in Solution. J. Am. Chem. Soc. 2007, 129, 2147-2160.

(17) Cannizzo, A.; Blanco-Rodriguez, A. M.; El Nahhas, A.; Sebera, J.; Zalis, S.; Vlcek, A.; Chergui, M. Femtosecond Fluorescence and Intersystem Crossing in Rhenium(I) Carbonyl-Bipyridine Complexes. J. Am. Chem. Soc. 2008, 130, 8967-8974.

(18) Cannizzo, A.; Milne, C. J.; Consani, C.; Gawelda, W.; Bressler, C.; van Mourik, F.; Chergui, M. Light-Induced Spin Crossover in $\mathrm{Fe}(\mathrm{II})$-based Complexes: The Full Photocycle Unraveled by Ultrafast Optical and X-ray Spectroscopies. Coord. Chem. Rev. 2010, 254, 26772686.

(19) Iwamura, M.; Watanabe, H.; Ishii, K.; Takeuchi, S.; Tahara, T. Coherent Nuclear Dynamics in Ultrafast Photoinduced Structural Change of Bis(diimine)copper(I) Complex. J. Am. Chem. Soc. 2011, 133, 7728-7736.

(20) Gawelda, W.; Cannizzo, A.; Pham, V. T.; van Mourik, F.; Bressler, C.; Chergui, M. Ultrafast Nonadiabatic Dynamics of [Fe$\mathrm{II}($ bpy) (3)](2+) in Solution. J. Am. Chem. Soc. 2007, 129, 8199-8206.

(21) Bram, O.; Messina, F.; El-Zohry, A. M.; Cannizzo, A.; Chergui, M. Polychromatic Femtosecond Fluorescence Studies of MetalPolypyridine Complexes in Solution. Chem. Phys. 2012, 393, 51-57.

(22) Kallioinen, J.; Benko, G.; Sundstrom, V.; Korppi-Tommola, J. E. I.; Yartsev, A. P. Electron Transfer from the Singlet and Triplet Excited States of $\mathrm{Ru}(\mathrm{dcbpy})(2)(\mathrm{NCS})(2)$ into Nanocrystalline $\mathrm{TiO}_{2}$ Thin Films. J. Phys. Chem. B 2002, 106, 4396-4404.

(23) Benko, G.; Kallioinen, J.; Korppi-Tommola, J. E. I.; Yartsev, A. P.; Sundstrom, V. Photoinduced Ultrafast Dye-to-Semiconductor Electron Injection From Nonthermalized and Thermalized Donor States. J. Am. Chem. Soc. 2002, 124, 489-493.

(24) Bram, O.; Cannizzo, A.; Chergui, M. Ultrafast Fluorescence Studies of Dye Sensitized Solar Cells. Phys. Chem. Chem. Phys. 2012, 14, 7934-7937.
(25) Wenger, B.; Gratzel, M.; Moser, J. E. Rationale for Kinetic Heterogeneity of Ultrafast Light-Induced Electron Transfer from $\mathrm{Ru}$ (II) Complex Sensitizers to Nanocrystalline $\mathrm{TiO}_{2}$. J. Am. Chem. Soc. 2005, 127, 12150-12151.

(26) Listorti, A.; O’Regan, B.; Durrant, J. R. Electron Transfer Dynamics in Dye-Sensitized Solar Cells. Chem. Mater. 2011, 23, 3381-3399.

(27) Pijpers, J. J. H.; Ulbricht, R.; Derossi, S.; Reek, J. N. H.; Bonn, M. Picosecond Electron Injection Dynamics in Dye-Sensitized Oxides in the Presence of Electrolyte. J. Phys. Chem. C 2011, 115, 2578-2584.

(28) Teuscher, J.; Decoppet, J. D.; Punzi, A.; Zakeeruddin, S. M.; Moser, J. E.; Gratzel, M. Photoinduced Interfacial Electron Injection Dynamics in Dye-Sensitized Solar Cells under Photovoltaic Operating Conditions. J. Phys. Chem. Lett. 2012, 3, 3786-3790.

(29) Sauve, G.; Cass, M. E.; Coia, G.; Doig, S. J.; Lauermann, I.; Pomykal, K. E.; Lewis, N. S. Dye Sensitization of Nanocrystalline Titanium Dioxide with Osmium and Ruthenium Polypyridyl Complexes. J. Phys. Chem. B 2000, 104, 6821-6836.

(30) Altobello, S.; Argazzi, R.; Caramori, S.; Contado, C.; Da Fre, S.; Rubino, P.; Chone, C.; Larramona, G.; Bignozzi, C. A. Sensitization of Nanocrystalline $\mathrm{TiO}_{2}$ with Black Absorbers Based on Os and $\mathrm{Ru}$ Polypyridine Complexes. J. Am. Chem. Soc. 2005, 127, 15342-15343.

(31) Verma, S.; Kar, P.; Das, A.; Palit, D. K.; Ghosh, H. N. Interfacial Electron-Transfer Dynamics on $\mathrm{TiO}_{2}$ and $\mathrm{ZrO}_{2}$ Nanoparticle Surface Sensitized by New Catechol Derivatives of Os(II)-Polypyridyl Complexes: Monitoring by Charge-Transfer Emission. J. Phys. Chem. C 2008, 112, 2918-2926.

(32) Verma, S.; Kar, P.; Das, A.; Palit, D. K.; Ghosh, H. N. The Effect of Heavy Atoms on Photoinduced Electron Injection from Nonthermalized and Thermalized Donor States of M-II-Polypyridyl $(\mathrm{M}=\mathrm{Ru} / \mathrm{Os})$ Complexes to Nanoparticulate $\mathrm{TiO} 2$ Surfaces: An Ultrafast Time-Resolved Absorption Study. Chem.-Eur. J. 2010, 16, 611-619.

(33) Wu, K. L.; Ho, S. T.; Chou, C. C.; Chang, Y. C.; Pan, H. A.; Chi, Y.; Chou, P. T. Engineering of Osmium(II)-Based Light Absorbers for Dye-Sensitized Solar Cells. Angew. Chem., Int. Ed. 2012, 51, 56425646.

(34) Kinoshita, T.; Fujisawa, J.; Nakazaki, J.; Uchida, S.; Kubo, T.; Segawa, H. Enhancement of Near-IR Photoelectric Conversion in Dye-Sensitized Solar Cells Using an Osmium Sensitizer with Strong Spin-Forbidden Transition. J. Phys. Chem. Lett. 2012, 3, 394-398.

(35) Maubert, B.; McClenaghan, N. D.; Indelli, M. T.; Campagna, S. Absorption Spectra and Photophysical Properties of a Series of Polypyridine Ligands Containing Appended Pyrenyl and Anthryl Chromophores and of their Ruthenium(II) and Osmium(II) Complexes. J. Phys. Chem. A 2003, 107, 447-455.

(36) Baldo, M. A.; O’Brien, D. F.; You, Y.; Shoustikov, A.; Sibley, S.; Thompson, M. E.; Forrest, S. R. Highly Efficient Phosphorescent Emission from Organic Electroluminescent Devices. Nature 1998, 395, 151-154.

(37) Chergui, M. On the Interplay Between Charge, Spin and Structural Dynamics in Transition Metal Complexes. Dalton Trans. 2012, 41, 13022-13029.

(38) van der Veen, R. M.; Cannizzo, A.; van Mourik, F.; Vlcek, A.; Chergui, M. Vibrational Relaxation and Intersystem Crossing of Binuclear Metal Complexes in Solution. J. Am. Chem. Soc. 2011, 133, 305-315.

(39) Steffen, A.; Tay, M. G.; Batsanov, A. S.; Howard, J. A. K.; Beeby, A.; Vuong, K. Q.; Sun, X. Z.; George, M. W.; Marder, T. B. 2,5-Bis(pR-arylethynyl)rhodacyclopentadienes Show Intense Fluorescence: Denying the Presence of a Heavy Atom. Angew. Chem., Int Ed. 2010, 49, 2349-2353.

(40) Braun, D.; Hensler, G.; Gallhuber, E.; Yersin, H. Vibronic and Magnetic Coupling in the Radiative Deactivation of the Lowest Excited-State of [Os(Bpy)3]2+ Doped into $[\mathrm{Ru}(\mathrm{Bpy}) 3](\mathrm{Pf} 6) 2$. J. Phys. Chem. 1991, 95, 1067-1073.

(41) Campagna, S.; Bartolotta, A.; Dimarco, G. Photophysics of $\mathrm{Ru}$ (II)-Polypyridine and Os(II)-Polypyridine Complexes in Poly(ethyleneoxide) Matrices. Chem. Phys. Lett. 1993, 206, 30-36. 
(42) Huber, P.; Yersin, H. Highly Resolved Emission of [Os(Bpy$\mathrm{H} 8) \mathrm{N}(\mathrm{Bpy}-\mathrm{D} 8) 3-\mathrm{N}] 2+(\mathrm{N}=0-3)$ - Evidence for Electronic Delocalization. J. Phys. Chem. 1993, 97, 12705-12709.

(43) Pentlehner, D.; Grau, I.; Yersin, H. Triplet State Properties of [Os(phen)(2)(dppene)](2+) in Different Host Materials and Host to Guest Energy Transfer in PVK. Chem. Phys. Lett. 2008, 455, 72-78.

(44) Verma, S.; Kar, P.; Banerjee, T.; Das, A.; Ghosh, H. N. Sequential Energy and Electron Transfer in Polynuclear Complex Sensitized $\mathrm{TiO}_{2}$ Nanoparticles. J. Phys. Chem. Lett. 2012, 3, 15431548.

(45) Vengris, M.; van der Horst, M. A.; Zgrablic, G.; van Stokkum, I. H. M.; Haacke, S.; Chergui, M.; Hellingwerf, K. J.; van Grondelle, R.; Larsen, D. S. Contrasting the Excited-State Dynamics of the Photoactive Yellow Protein Chromophore: Protein Versus Solvent Environments. Biophys. J. 2004, 87, 1848-1857.

(46) Zgrablic, G.; Voitchovsky, K.; Kindermann, M.; Haacke, S.; Chergui, M. Ultrafast Excited State Dynamics of the Protonated Schiff Base of All-Trans Retinal in Solvents. Biophys. J. 2005, 88, 2779-2788.

(47) Cannizzo, A.; Bram, O.; Zgrablic, G.; Tortschanoff, A.; Oskouei, A. A.; van Mourik, F.; Cherguil, M. Femtosecond Fluorescence Upconversion Setup with Broadband Detection in the Ultraviolet. Opt. Lett. 2007, 32, 3555-3557.

(48) Kalyanasundaram, K.; Nazeeruddin, M. K. Photophysical and Redox Properties of Mono-Nuclear and Bi-Nuclear Complexes of Osmium(II) with 2,3-Bis(2-Pyridyl)Pyrazine as Bridging Ligand. Chem. Phys. Lett. 1989, 158, 45-50.

(49) Nazeeruddin, M. K.; Zakeeruddin, S. M.; Kalyanasundaram, K. Enhanced Intensities of the Ligand-to-Metal Charge-Transfer Transitions in $\mathrm{Ru}(\mathrm{III})$ and $\mathrm{Os}(\mathrm{III})$ Complexes of Substituted Bipyridines. J. Phys. Chem. 1993, 97, 9607-9612.

(50) Barigelletti, F.; Decola, L.; Balzani, V.; Hage, R.; Haasnoot, J. G.; Reedijk, J.; Vos, J. G. Mononuclear and Dinuclear Osmium(Ii) Compounds Containing 2,2'-Bipyridine and 3,5-Bis(pyridin-2-yl)1,2,4-triazole - Synthesis, Electrochemistry, Absorption-Spectra, and Luminescence Properties. Inorg. Chem. 1991, 30, 641-645.

(51) Hankache, J.; Niemi, M.; Lemmetyinen, H.; Wenger, O. S. Photoinduced Electron Transfer in Linear Triarylamine-Photosensitizer-Anthraquinone Triads with Ruthenium(II), Osmium(II), and Iridium(III). Inorg. Chem. 2012, 51, 6333-6344.

(52) Yersin, H.; Strasser, J. Triplets in Metal-Organic Compounds. Chemical Tunability of Relaxation Dynamics. Coord. Chem. Rev. 2000, 208, 331-364. 